\title{
Terneza, grasa intramuscular y de cobertura en carne de novillos faenados en Corrientes (Argentina)
}

\author{
Ynsaurralde, A.E. ${ }^{1}$, Rébak, G.I. ${ }^{2}$; Sánchez, S. ${ }^{2}$; Capellari, A. ${ }^{2}$ \\ ${ }^{1}$ Instituto Nacional de Tecnología Agropecuaria (INTA Mercedes, Corrientes, Argentina). \\ ${ }^{2}$ Facultad de Ciencias Veterinarias, Universidad Nacional del Nordeste, UNNE, \\ Sargento Cabral 2139, Corrientes (3400), Argentina. \\ E-mail: ynsaurralde.eugenia@inta.gob.ar
}

\begin{abstract}
Resumen
Ynsaurralde, A.E.; Rébak, G.I.; Sánchez, S.; Capellari, A.: Terneza, grasa intramuscular y de cobertura en carne de novillos faenados en Corrientes (Argentina). Rev. vet. 24: 2, 86-90, 2013. La terneza de la carne es el atributo más apreciado por los consumidores, encontrándose condicionada por muchos factores. El objetivo de este trabajo fue generar información sobre terneza objetiva post maduración de la carne bovina y determinar valores de grasa intramuscular y de cobertura en animales de diferentes biotipos y edades faenados en Corrientes. El trabajo se realizó en un frigorífico tipo A y en la Facultad de Ciencias Veterinarias de la UNNE. Se evaluó el músculo longisimus dorsi de novillos tipo Brangus y Braford, de 4, 6 y 8 dientes. Se registró el peso individual de res caliente, así como la conformación y terminación. Las muestras divididas en dos fueron maduradas durante 7 y 14 días envasadas al vacío. La terneza se evaluó por la cizalla de Warner-Bratzler, la grasa total por el método de Soxhlet y la grasa de cobertura con un escalímetro. Se utilizó el análisis de la covarianza a tres vías incluyendo el peso de la res como covariable. El periodo de maduración afectó la terneza. Se registraron diferencias estadísticas entre tratamientos a los 14 días, no así a los 7 días. Durante este periodo, la diferencia de peso lograda se atribuyó al número de dientes y la covariable. El espesor de grasa dorsal se vio afectado por el número de dientes al igual que en la grasa intramuscular, donde además afectó la covariable. La maduración al vacío mejoró la terneza de la carne, siendo este efecto manifiesto en individuos más jóvenes, incrementándose la grasa de cobertura con la edad, no así la grasa intramuscular. La carne producto de animales faenados en la Provincia de Corrientes debe ser considerada de buena calidad, ya que según características de terneza y porcentajes de grasa intramuscular y de cobertura encontradas, responde a las más altas exigencias del mercado.
\end{abstract}

Palabras clave: novillo, maduración de la carne, grasa, terneza, edad, tipo genético.

\begin{abstract}
Ynsaurralde, A.E.; Rébak, G.I.; Sánchez, S.; Capellari, A.: Tenderness, intramuscular and covering fat in meat of steers slaughtered in Corrientes (Argentina). Rev. vet. 24: 2, $86-90,2013$. The tenderness of meat is the most valued attribute for the market, being influenced by many factors. The aims of this study were to generate information on post objective tenderness values of beef maturation and to estimate both intramuscular and cover fat values in animals of different ages and biotypes slaughtered in Corrientes, Argentina. Assay was performed in a type A slaughterhouse and at the Faculty of Veterinary Science UNNE. Longisimus dorsi muscle of 4, 6 and 8 teeth Brangus and Braford steers was evaluated. Individual weight, conformation and termination on the hot carcass were registered. The samples were divided in two groups vacuum packed, maturated for 7 and 14 days. Tenderness was assessed by Warner-Bratzler shear, total fat by the Soxhlet method, and fat coverage with a scaler. We used analysis of covariance for three tracks including the carcass weight as covariable. The period of maturation affected tenderness. There were statistical differences between treatments at 14 days, but not at 7 . During this period, the difference of weight achieved was attributable to number of teeth and the covariable. The back fat thickness was affected by the number of teeth as in intramuscular fat which also affected the covariable. Vacuum maturation accentuated the tenderness of beef, being more evident in younger individuals, with cover fat increasing according age; on the contrary, this effect was not observed for intramuscular fat. Meat products from animals slaughtered in Corrientes must be consid-
\end{abstract}

Recibido: 27 junio 2013 / Aceptado: 4 julio 2013

Tesis Maestría Producción Animal Subtropical, FCV-UNNE 
ered of very good quality, according to the characteristics of tenderness and intramuscular as well as cover fat percentages that meet high market demands.

Key words: steer, meat maturation, fat, tenderness, age, genetic type.

\section{INTRODUCCIÓN}

Argentina es considerada a nivel mundial como país productor de alimentos, especialmente de granos y carne, dado el gran volumen de comercialización a países extranjeros. La calidad de la producción y la técnica de comercialización de carne bovina son factores importantes para determinar la inserción en el mercado, principalmente el externo. Uno de los problemas que presenta la carne argentina es la inconsistencia en la terneza, ya que se la produce a partir de diferentes biotipos y condiciones ${ }^{20}$.

La terneza es afectada por un gran número de factores, entre ellos la genética, la edad, el sexo, la alimentación y el manejo pre y post faena de los animales, como ser presencia de situaciones estresantes durante la carga, el viaje o previos al sacrificio, como también factores relacionados con el manejo del frío durante la transformación del músculo en carne y maduración de la misma ${ }^{21}$.

En la región subtropical de Argentina predominan razas como el Braford, Brangus y en menor escala Santa Gertrudis ${ }^{12}$ originadas a partir del Bos indicus, en cuyo músculo se han registrado elevados niveles de actividad de calpastatina ${ }^{24,25}$ enzima que inhibe a la calpaina, responsable de la tiernización de la carne ${ }^{14}$. A su vez, la terneza disminuye al aumentar la edad del animal, debido a que las moléculas de tropocolágeno forman enlaces cruzados intermoleculares que confieren al colágeno su firmeza ${ }^{27}$.

A medida que los animales envejecen los enlaces cruzados se estabilizan y el diámetro medio de las fibrillas aumenta. Un alto plano nutricional y un rápido crecimiento (invernadas intensivas, feed lots) provocan un alto índice de síntesis de colágeno más inestable, resultando de esta forma un músculo con mayor terneza. Además, dietas con un elevado contenido energético, proporcionan carne más tierna, debido probablemente al mayor contenido de grasa infiltrada ${ }^{10}$. La presencia de grasa entre la porción magra del músculo (veteado o marmorización), junto a la capacidad de retención de agua, contribuyen a la jugosidad y al aroma de la carne fresca ${ }^{8,27}$, además de estimular la secreción de saliva y lubricar las fibras musculares durante el cocinado ${ }^{10}$.

El presente trabajo se basó en la hipótesis que la maduración incrementa la terneza de la carne vacuna, estando la grasa intramuscular y de cobertura afectadas por el biotipo y la edad de faena de los novillos. En tal sentido, los objetivos fueron generar información sobre valores de terneza objetiva post maduración en cortes envasados al vacío y determinar porcentajes de grasa intramuscular y espesor de grasa de cobertura presentes en el músculo longissimus dorsi de animales de diferentes biotipos y edades sacrificados en Corrientes (Argentina).

\section{MATERIAL Y MÉTODOS}

Las actividades se llevaron a cabo en un frigorífico tipo A de la localidad de Riachuelo y en el laboratorio de Tecnología de los Alimentos de la Facultad de Ciencias Veterinarias de la UNNE (Corrientes, Argentina).

$\mathrm{Se}$ evaluaron novillos con destino a diferentes mercados internacionales, con un peso vivo superior a $400 \mathrm{~kg}$, de 4, 6 y 8 dientes. En la playa de faena se seleccionaron los animales a utilizar, consignándose la procedencia y el destino comercial, identificando los ejemplares por número de garrón y biotipo, clasificándolos en tipos Brangus y Braford según aspectos externos. La edad aproximada se determinó por cronometría dentaria (4, 6 y 8 dientes sin nivelar). En el palco de tipificación y clasificación se registraron los pesos individuales de las reses calientes.

Las carcasas fueron depositadas en cámara frigorífica por el término de $24 \mathrm{~h}$ como mínimo hasta alcanzar la temperatura óptima de despostada $\left(7^{\circ} \mathrm{C}\right.$ o menos) y un $\mathrm{pH}$ inferior a 5,9. Posteriormente fueron seccionadas a nivel de la séptima costilla con sierra circular en cuarto delantero, trasero y costillar, donde se procedió a la toma de muestras del músculo longisimus dorsi entre la $11^{\mathrm{a}}$ y $13^{\mathrm{a}}$ costillas, con un espesor mínimo de 2,5 $\mathrm{cm}$. Las muestras fueron subdivididas en dos submuestras y acondicionadas en bolsas de vacío multilaminadas, identificándolas con tarjetas de alto impacto, procediéndose a efectuar el vacío y termo-contraído.

Trasladadas al laboratorio, las muestras se conservaron refrigeradas $\left(4^{\circ} \mathrm{C}\right)$ hasta alcanzar los 7 días de maduración para la submuestra 1 y 14 días para la submuestra 2. Alcanzados estos períodos, se procedió a la congelación a $-20^{\circ} \mathrm{C}$ para detener el proceso de maduración, hasta el momento del procesamiento. La terneza (fuerza de corte) se determinó por la cizalla de Warner-Bratzler, para ello las muestras fueron descongeladas $24 \mathrm{~h}$ en refrigeración y cocidas $1 \mathrm{~h}$ en baño maría a $70^{\circ} \mathrm{C}$. Se evaluaron como mínimo cinco muestras de $1,27 \mathrm{~cm}$ de diámetro. La grasa total se determinó por el extractor de Soxhlet ${ }^{1}$. La grasa de cobertura se midió con un escalímetro, en una línea ubicada en las tres cuartas partes externas del bife.

Los valores de terneza se analizaron según periodo de maduración, biotipo y número de dientes con las interacciones correspondientes según un análisis de co- 
variancia a tres vías incluyendo el peso de la res como co-variable, según un diseño experimental con arreglo factorial $2 \times 2 \times 3$, incluyendo todas las interacciones posibles. Posteriormente se analizaron a los 7 y 14 días mediante el análisis de la co-varianza a dos vías, según un diseño experimental con arreglo factorial $2 \times 3$, evaluando la presencia de interacción e incluyendo como efectos principales la edad y el genotipo, y el peso de la res como co-variable. Los valores de grasa intramuscular y de cobertura se analizaron mediante el modelo estadístico utilizado en la evaluación de la terneza a los 7 y 14 días. Todas las comparaciones de medias $a$ posteriori se realizaron con el test de Duncan.

\section{RESULTADOS Y DISCUSIÓN}

El peso de carcasa aumentó significativamente con la edad: los individuos de 8 dientes resultaron más pesados que los de 4 dientes. Se encontró efecto significativo del periodo de maduración sobre la terneza de la muestras $(p<0,05)$ sin presentarse interacciones significativas entre las mismas (Tabla 1).

Mediante los análisis anteriores se evidenció el efecto del período de maduración sobre los valores de fuerza de corte analizados, resultados similares a los obtenidos por otros autores, quienes corroboraron que la terneza se incrementa al aumentar el tiempo de almacenamiento ${ }^{28}$, debido a la actividad enzimática causante de cambios ultraestructurales en el sarcómero ${ }^{14}$ . Esto genera la necesidad de analizar los períodos de maduración de manera independiente a fin de observar el comportamiento de los datos e identificar efectos significativos de las variables clasificatorias.

Alos 7 días de maduración, no se observaron diferencias estadísticamente significativas de terneza entre tratamientos $(p>0,05)$. En otras investigaciones se encontraron valores de fuerza de corte similares a los logrados en esta experiencia, en novillos raza Holando y Blue Belga de 28 meses de edad ${ }^{9,15}$, mientras que otros registraron valores superiores en toros Rojos Noruegos con 9 días de maduración y en hembras Brahman x Philippine Native con $6,83 \mathrm{~kg}$ y $6,26 \mathrm{~kg}$ respectivamente ${ }^{11,17}$.

A los 14 días de maduración, se observaron diferencias estadísticamente significativas para el número de dientes $(\mathrm{p}<0,05)$, sin detectarse significación para el factor biotipo, la interacción o la covariable $(\mathrm{p}>0,05)$ (Tabla 2). Al igual que en otros estudios, no se registraron diferencias entre individuos de 4 y 6 dientes ${ }^{22}$, siendo nuestros valores similares a los informados por algunos autores ${ }^{3}$ y superiores a los obtenidos por otros en novillos cruza Bonsmara x Beefmaster ${ }^{2}$.

Para las modificaciones estimadas en la terneza entre los 7 y 14 días de maduración se observaron diferencias estadísticamente significativas para el número de dientes así como para la covariable peso de carcasa $(p<0,05)$, sin detectarse significación para el factor biotipo y la interacción ( $>00,05)$ (Tabla 2). Según los resultados descriptos, la edad cronológica (representa-
Tabla 1. Determinación de la terneza objetiva en carne de novillos según período de maduración.

\begin{tabular}{lccc}
\hline maduración & $\mathrm{n}$ & $\overline{\mathrm{x}} \pm \mathrm{DE}$ & $\mathrm{p}$ \\
\hline 7 días & 132 & $3,66 \mathrm{a} \pm 1,06$ & $<0,05$ \\
14 días & 132 & $3,35 \mathrm{~b} \pm 0,83$ & \\
\hline
\end{tabular}

$\overline{\mathrm{x}}$ : media aritmética, DE: desvío estándar, p: significancia. Letras distintas indican diferencias significativas.

Tabla 2. Valores de terneza objetiva con 7 y 14 días de maduración y sus diferencias.

\begin{tabular}{lcccc}
\hline maduración & $\mathrm{N}^{\circ}$ dientes & $\mathrm{n}$ & $\overline{\mathrm{x}} \pm \mathrm{DE}$ & $\mathrm{p}$ \\
\hline \multirow{4}{*}{14 días } & 4 & 43 & $3,17 \pm 0,80 \mathrm{~b}$ & \\
& 6 & 43 & $3,22 \pm 0,74 \mathrm{~b}$ & $<0,05$ \\
& 8 & 46 & $3,63 \pm 0,89 \mathrm{a}$ & \\
\multirow{4}{*}{$14 / 7$ días } & 4 & 43 & $0,57 \pm 1,11 \mathrm{a}$ & \\
& 6 & 43 & $0,32 \pm 0,70 \mathrm{ab}$ & $<0,05$ \\
& 8 & 46 & $0,06 \pm 0,81 \mathrm{~b}$ & \\
\hline
\end{tabular}

$\overline{\mathrm{X}}$ : media aritmética, DE: desvío estándar, p: significancia. Letras distintas indican diferencias significativas.

Tabla 3. Valores de grasa de cobertura y grasa total.

\begin{tabular}{lcccc}
\hline grasa & $\mathrm{N}^{\mathrm{o}}$ dientes & $\mathrm{n}$ & $\overline{\mathrm{x}} \pm \mathrm{DE}$ & $\mathrm{p}$ \\
\hline \multirow{3}{*}{ cobertura } & 4 & 15 & $8,04 \pm 4,44 \mathrm{~b}$ & \\
& 6 & 18 & $10,63 \pm 3,41 \mathrm{ab}$ & $<0,05$ \\
& 8 & 27 & $12,04 \pm 4,59 \mathrm{a}$ & \\
\multirow{2}{*}{ total } & 4 & 24 & $2,79 \pm 1,42$ & \\
& 6 & 23 & $2,17 \pm 0,98$ & $>0,05$ \\
& 8 & 30 & $3,09 \pm 1,68$ & \\
\hline
\end{tabular}

$\overline{\mathrm{X}}$ : media aritmética, DE: desvío estándar, p: significancia. Letras distintas indican diferencias significativas.

da aquí por el número de dientes) tiene efecto sobre la tiernización de la carne tras la maduración, coincidiendo estos guarismos con los de investigadores que aseveran que la edad de faena del animal correlaciona significativamente con la fuerza de corte, encontrando un efecto lineal en la misma ${ }^{5,28}$.

Así, las menores diferencias de terneza encontradas entre los 7 y 14 días en los animales de 8 dientes pueden ser atribuidas a su mayor edad, debido a que con la misma aumenta la cantidad de enlaces cruzados entre moléculas de colágeno, disminuyendo su solubilidad ${ }^{26}$, sin registrarse efecto de la maduración sobre dicha fracción ${ }^{18,23}$. El tiempo de maduración ejerce un rol preponderante en la tiernización de la carne ${ }^{16,28}$ . Así, muestras sin maduración arrojaron valores de terneza inferiores a los aquí obtenidos, como los registrados en novillos Braford $(4,36 \mathrm{~kg})$ y Criollo $(5,39 \mathrm{~kg}){ }^{19}$.

En novillos Brahman se obtuvieron valores de modificación de la terneza entre los días 7 y 14 de maduración similares a los presentados en este trabajo, de $0,61 \mathrm{~kg}^{25}$, mientras que en novillos británicos y cruzas continentales, se encontró una mayor respuesta entre los días 14 y $21(0,4 \mathrm{~kg})$ que entre 7 y $14(0,2 \mathrm{~kg})^{4}$. 
En grasa de cobertura, se registró efecto del número de dientes $(\mathrm{p}<0,05)$, no así para el biotipo, la interacción o la covariable $(\mathrm{p}>0,05)$. Para grasa intramuscular solo se registró efecto de la covariable $(p<0,05)$ (Tabla 3). Considerando que el valor de $\mathrm{p}$ encontrado para el efecto diente fue muy cercano a la significación, se aplicó el test de Duncan, encontrándose diferencias significativas. Los resultados arrojaron una menor cantidad de grasa intramuscular en individuos faenados con 6 dientes y los mayores valores en los de 8 dientes, en ambos biotipos, evidenciándose efecto de la edad. Esto puede explicarse ya que la cantidad de proteína de un animal crece en forma casi lineal y es bastante predecible, pero la grasa es muy variable porque se incrementa en forma exponencial con el aumento de peso y con la tasa de ganancia de peso ${ }^{7}$.

En novillos Braford recriados y terminados sobre pasturas tropicales, de 2,5 años y $212,57 \mathrm{~kg}$ de peso de carcasa promedio, se obtuvieron valores similares a los presentados en este trabajo de grasa intramuscular $(2,73 \%)$ e inferiores de grasa de cobertura $(2,98 \mathrm{~mm}){ }^{19}$. Valores similares de grasa intramuscular se registraron en Brangus terminados sobre concentrados; este autor registró valores inferiores en otras razas estudiadas en el mismo trabajo $(1,64 \%$ en Limousin y $0,64 \%$ en Blue Belga ${ }^{6}$. En novillos Blue Belga se registraron pesos de carcasa de $370 \mathrm{~kg}$, muy superiores a los nuestros, con un valor de grasa intramuscular similar $(2,4 \%)^{15}$.

En novillos Bonsmara x Beefmaster terminados en feedlot se registraron valores inferiores de grasa de cobertura $(7 \mathrm{~mm})^{2}$. Otros investigadores, en novillos de 15 meses de diversas cruzas Angus con Bos indicus, mantenidos post destete en pasturas y terminados sobre maíz, encontraron niveles de grasa de cobertura similares a los nuestros, de $12,7 \mathrm{~mm}$ de promedio ${ }^{13}$, al igual que en novillos Brahman con 12,56 mm ${ }^{25}$.

En conclusión, la maduración afecta la calidad de la carne vacuna ya que aumenta la terneza de la misma, siendo este efecto más manifiesto en individuos jóvenes. La grasa de cobertura se incrementa con la edad, no así la grasa intramuscular. La carne producto de animales faenados en la Provincia de Corrientes y destinados a exportación, debe ser considerada de buena calidad, ya que según sus características de terneza y grasa intramuscular y de cobertura responde a las más altas exigencias del mercado.

Agradecimiento. Al Frigorífico Tomas Arias S.A. por permitirnos el ingreso a la planta y ceder gentilmente las muestras de carne.

\section{REFERENCIAS}

1. Association of Official Analytical Chemist (AOAC). 2000. Research guidelines for cookery, sensory evaluation and instrumental tenderness measurements of fresh meat, Publ. AOAC, 17th ed, Arlington (USA), 186 p.

2. Behrends SM, Miller RK, Rouquette FM, Randel RD, Warrington BG, Forbes TD, Welsh TH, Lippke H, Beh- rends JM, Carstens GE, Holloway JE. 2009. Relationship of temperament, growth, carcass characteristics and tenderness in beef steers. Meat Sci 81: 433-438.

3. Belew JB, Brooks JC, McKenna DR, Savell JW. 2003. Warner-Bratzler shear evaluations of 40 bovine muscles. Meat Scie 64: 507-512.

4. Claus HL, Dikeman ME, Murray L, Brooks JC, Shook J, Hilton GG, Lawrence TE, Mehaffey JM, Johnson BJ, Allen DM, Streeter MN, Nichols WT, Hutcheson JP, Yates DA, Miller MF, Hunt MC, Killefer J. 2010. Effects of supplementing feedlot steers and heifers with zilpaterol hydrochloride on Warner-Bratzler shear force interrelationships of steer and heifer longissimus lumborum and heifer triceps brachii and gluteus medius muscles aged for 7, 14 and 21 d. Meat Sci 85: 347-355.

5. Cross HR, Carpenter ZL, Smith GC. 1973. Effects of intramuscular collagen and elastin on bovine muscle tenderness. J Food Sci 38: 998-1003.

6. Cuvelier C, Clinquart A, Hocquette JF, Cabaraux JF, Dufrasne I, Istasse JL. 2006. Comparison of composition and quality traits of meat from young finishing bulls from Belgian Blue, Limousin and Aberdeen Angus breeds. Meat Sci 74: 522-531.

7. Di Marco O. 2006. Crecimiento de vacunos para carne, Ed. INTA Balcarce (Argentina), 204 p.

8. Forrest J, Aberle E, Judge M, Merkel R. 1979. Fundamentos de la ciencia de la carne, $2^{\circ}$ ed., Acribia, Zaragoza, p. 356 .

9. Franco J, Feed O, Garibotto G, Ballesteros F, Forichi F, Bentancur O, Bianchi G. 2008. Efecto del "tendercut", vitamina D3 y maduración sobre la textura y calidad sensorial de la carne vacuna. Rev Arg Prod Anim 28: 45-52.

10. Garcia MD. 2000. Metodología para el estudio de la calidad de la canal y de la carne en rumiantes, Publ. Inst Nac Invest Tecnol Agr, Madrid, p. 9-16.

11. Hildrum KI, Rødbotten R, Høy M, Berg J, Naruma B, Wold JP. 2009. Classification of different bovine muscles according to sensory characteristics and Warner Bratzler shear force. Meat Sci 83: 302-307.

12. Instituto Nacional de Tecnología Agropecuaria (INTA). 2007. La producción de carne en Argentina. Programa Nacional de Carnes, Publ. INTA, Buenos Aires, p.19.

13. King DA, Morgan WW, Miller RK, Sanders JO, Lunt DK, Taylor JF, Gill CA, Savell JW. 2006. Carcass merit between and among family groups of Bos indicus crossbred steers and heifers. Meat Sci 72: 496-502.

14. Koohmaraie M, Geesink GH. 2006 Contribution of postmortem muscle biochemistry to the delivery of consistent meat quality with particular focus on the calpain system. Meat Scie 74: 34-43.

15. Maher SC, Mullen AM, Buckley DJ, Kerry JP, Moloney AP. 2005. The influence of biochemical differences on the variation in tenderness of M. longissimus dorsi of Belgian Blue steers managed homogenously pre and postslaughter. Meat Sci 69: 215-224.

16. María GA, Villarroel M, Sanudoa C, Olletaa JL, Gebresenbet G. 2003. Effect of transport time and ageing on aspects of beef quality. Meat Sci 65: 1335-1340. 
17. Neath KE, Del Barrio AN, Lapitan RM, Herrera JV, Cruz JL, Fujihara T, Muroya S, Chikuni K, Hirabayashi M, Kanai Y. 2007. Difference in tenderness and $\mathrm{pH}$ decline between water buffalo meat and beef during postmortem aging. Meat Sci 75: 499-505.

18. Ngapo TM, Berge P, Culioli J, Dransfield E, De Smet S, Claeys E. 2002. Perimysial collagen crosslinks and meat tenderness in Belgian Blue double-muscled cattle. Meat Sci 61: 91-102.

19. Orellana C, Peña F, García A, Perea J, Martos J, Domenech V, Acero R. 2009. Carcass characteristics, fatty acid composition, and meat quality of Criollo Argentino and Braford steers raised on forage in a semi-tropical region of Argentina. Meat Sci 81: 57-64.

20. Papaleo J. 2006. Efectos genéticos y de la maduración sobre calidad de la carne bovina, Tesis MSci, INTA, Unidad Integrada Balcarce (Argentina), p. 1.

21. Peluffo Frisco M, Monteiro M. 2002. Terneza: una característica a tener en cuenta. Instituto Plan Agropecuario Uruguay. On line: www.planagro.com.uy, p. 4.

22. Pflanzer SB, de Felicio PE. 2009. Effects of teeth maturity and fatness of Nelore (Bos indicus) steer carcasses on instrumental and sensory tenderness. Meat Sci 83: 697 701 .
23. Pierson CJ, Fox, JD. 1976. Effect of postmortem aging time and temperature on $\mathrm{pH}$, tenderness and soluble collagen fractions in bovine Longissimus muscle. J Anim Sci 43: 1206-1210.

24. Pringle TD, Williams SE, Lamb BS, Johnson DD, West RL. 1997. Carcass characteristics, the calpain proteinase system, and aged tenderness of Angus and Brahman crossbred steers. J Anim Sci 75: 2955-2961.

25. Riley DG, Johnson DD, Chase CC, West RL, Coleman SW, Olson TA, Hammond AC. 2005. Factors influencing tenderness in steaks from Brahman cattle. Meat Sci 70: 347-356.

26. Torrescano G, Sanchez A, Giménez B, Roncales P, Beltran JA. 2003. Shear values of raw samples of 14 bovine muscles and their relation to muscle collagen characteristics. Meat Sci 64: 85-91.

27. Warris PD. 2003. Ciencia de la carne, Acribia, Zaragoza, p. 278.

28. Xiong YL, Mullins OE, Stika JF, Chen J, Blanchard S, Moody WG. 2007. Tenderness and oxidative stability of post-mortem muscles from mature cows of various ages. Meat Sci 77: 105-113. 